\title{
Good deals in markets with friction
}

\author{
ALEJANDRO BALBÁS*†, BEATRIZ BALBÁS $\ddagger$ and RAQUEL BALBÁS§ \\ †University Carlos III of Madrid, C/ Madrid 126, 28903 Getafe, Madrid, Spain \\ †University of Castilla La Mancha, Avda. Real Fábrica de Seda s/n, 45600 Talavera, Toledo, Spain \\ $\S$ Department of Actuarial and Financial Economics, University Complutense of Madrid, Somosaguas, 28223 Pozuelo de \\ Alarcón, Madrid, Spain
}

(Received 3 March 2011; in final form 21 February 2013)

\begin{abstract}
This paper studies an optimization problem involving pay-offs of (perhaps dynamic) investment strategies. The pay-off is the decision variable, the expected pay-off is maximized and its risk is minimized. The pricing rule may incorporate transaction costs and the risk measure is continuous, coherent and expectation bounded. We will prove the necessity of dealing with pricing rules such that there exists an essentially bounded stochastic discount factor that must also be bounded from below by a strictly positive value. Otherwise, good deals will be available to traders, i.e. depending on the selected risk measure, investors can choose pay-offs whose (risk, return) will be as close as desired to $(-\infty, \infty)$ or $(-1, \infty)$. This pathological property still holds for vector risk measures (i.e. if we minimize a vector-valued function whose components are risk measures). It is worth pointing out that, essentially, bounded stochastic discount factors are not usual in the financial literature. In particular, the most famous frictionless, complete and arbitrage-free pricing models imply the existence of good deals for every continuous, coherent and expectation bounded (scalar or vector) measure of risk, and the incorporation of transaction costs will not guarantee the solution of this caveat.
\end{abstract}

Keywords: Risk measures; Transaction costs; Portfolio optimization; Arbitrage relationship

JEL Classification: G1, G11, G12, G13

\section{Introduction}

Since Artzner et al. (1999) introduced the "coherent measures of risk' there has been growing interest in risk measures beyond the variance, and many authors have extended the discussion. Among many other interesting contributions, Föllmer and Schied (2002) defined the convex risk measures, Goovaerts et al. (2004) introduced the consistent risk measures, Rockafellar et al. (2006) defined the expectation bounded risk measures, Zhiping and Wang (2008) presented the two-sided coherent risk measures, Brown and Sim (2009) introduced the satisfying measures, and Aumann and Serrano (2008) and Foster and Hart (2009) defined indexes of riskiness. All of these measures are being used more and more by researchers, practitioners, regulators and supervisors.

Actuarial and financial applications of risk measures are continuously being developed. Interesting examples are portfolio theory and equilibrium (Rockafellar et al. 2007, Miller and Ruszczynski 2008, among others), pricing issues (Hamada and Sherris 2003, Staum 2004, Goovaerts and Laeven 2008, among others), optimal reinsurance (Centeno and Simoes

*Corresponding author. Email: alejandro.balbas@uc3m.es
2009, Dimitrova and Kaishev 2010, Guerra and Centeno 2012, among others), etc.

The notion of a 'good deal' was introduced by Cochrane and Saa-Requejo (2000). Mainly, a good deal is an investment strategy providing traders with a 'very high return/risk ratio', in comparison with the value of this ratio for the market portfolio. Risk is measured by means of the standard deviation, and the absence of a good deal is imposed in an arbitrage-free model so as to price in incomplete markets. This line of research has been extended for more general risk functions (see Staum (2004) or Arai (2011), amongst many other interesting contributions).

In addition, some recent papers deal with pricing models and risk measures and impose conditions that are strictly stronger than the absence of arbitrage. For instance, Stoica and Lib (2010) fix a pricing model and a risk measure such that the risk measure sub-gradient contains at least one equivalent riskneutral Probability Measure of the pricing model.

However, the fulfillment of these assumptions, stronger than the absence of arbitrage, is not so obvious in very important pricing models of financial economics. Balbás et al. (2010) have shown the existence of 'pathological results' when combining some risk measures ( $\mathrm{VaR}, \mathrm{CVaR}$, dual power transform (DPT), etc.) and very popular pricing models (Black and Scholes, Heston, etc.). Indeed, for the above examples, the 
stochastic discount factor (SDF) of the pricing model and the risk measure sub-gradient do not satisfy several conditions, which implies the existence of sequences of portfolios whose expected returns tend to plus infinity and whose risk levels tend to minus infinity or remain bounded (risk $=-\infty$ and return $=$ $+\infty$, or bounded risk and return $=+\infty$ ). In this paper we will use the expression 'good deal' to represent those sequences making the managers as rich as desired and obviously outperforming the market portfolio.

Needless to say that the existence of these good deals is a meaningless finding from a financial point of view, and is not supported by the empirical evidence. A possible solution could be the incorporation of friction, which could make traders lose many potential earnings. The objective of this paper is to analyse the existence of good deals in the presence of transaction costs, even when trading the riskless asset.

The outline of the article is as follows. Section 2 summarizes the basic properties of the risk measures and the imperfect pricing rules we deal with. We draw on a slight extension of the representation theorem of expectation bounded risk measures of Rockafellar et al. (2006), and the most important result of this section is a new representation theorem of the pricing rule (corollary 2.4). choice problem that minimizes the portfolio risk for every desired expected return. The absence of a good deal holds if the portfolio choice problem is bounded and the optimal risk increases, as does the required expected return. Both a primal and a dual approach are given, and the most important result is theorem 3.3, which guarantees the absence of a duality gap between both problems. The representation of probability measures on convex sets by points, a classic problem for Choquet integrals (Phelps 2001), and corollary 2.4 play a critical role in the proof of theorem 3.3. In other words, the absence of a duality gap is implied by three representation theorems: The first deals with risk measures, the second is related to pricing rules, an the third is for probability measures on convex sets.

Section 4 deals with the main problem of this paper, which is the absence or existence of a good deal under friction. The main results are theorem 4.3 and its remarks. They give the necessary and necessary and sufficient conditions to prevent the existence of a good deal. In particular, some SDF of the pricing rule must be a convex combination of the risk measure sub-gradient and the riskless asset, and the weight of the riskless asset must be strictly positive. It is also remarkable that most of the necessary conditions do not affect the risk measure, and only the pricing rule is involved. We will prove that pricing rules without essentially bounded SDF will provide traders with good deals for every risk measure that may be extended to the whole space $L^{1}$ ( $\mathrm{CVaR}$, for instance), and pricing rules without SDF bounded from below by a strictly positive value will provide traders with good deals regardless of the continuous, coherent and expectation bounded risk measures they use. These properties still hold for vector risk measures (i.e. vector-valued functions whose components are risk measures). Thus, the existence of $0<b \leq B$ and a SDF $z$ such that $b \leq z \leq B$ must hold. It is worth remarking that bounded SDF are not at all usual in the financial literature. In particular, pricing rules having a unique SDF (i.e. perfect markets) with the log-normal (Black and Scholes) or heavier-tailed (stochastic volatility pricing models)
Section 3 is devoted to the introduction of a general portfolio distribution will generate good deals for every continuous, coherent and expectation bounded (scalar or vector) risk measure.

Although transaction costs are presented in section 2 in a very general setting, additional constraints could be considered. This is the focus of section 5. It will be shown that the presence of quite natural cone constraints might partially solve the caveat we found in theorem 4.3. Section 6 presents the most important conclusions of the paper.

\section{Preliminaries and notation}

\subsection{The risk measure}

Consider the probability space $(\Omega, \mathcal{F}, \mathbb{P})$ composed of the set of 'states of the world' $\Omega$ that may occur within the time interval $[0, T]$, the $\sigma$-algebra $\mathcal{F}$ and the probability measure $\mathbb{P}$. If $p \in[1, \infty), L^{p}$ will denote the space of $\mathbb{R}$-valued random variables $y$ on $\Omega$ such that $\mathbb{E}\left(|y|^{p}\right)<\infty$, with $\mathbb{E}()$ representing the mathematical expectation. If $q \in(1, \infty]$ is its conjugate value (i.e. $1 / p+1 / q=1$ ), then the Riesz Representation Theorem (Rudin 1987, theorem 6.17, p. 127) guarantees that $L^{q}$ is the dual space of $L^{p}$, where $L^{\infty}$ is composed of essentially bounded random variables. A special important case arises for $p=q=2$.

Let $p \in[1,2]$ and $q \in[2, \infty]$, then

$$
\rho: L^{p} \longrightarrow \mathbb{R}
$$

will be the general risk function that a trader uses in order to control the risk level of his wealth at $T$. Denote by

$$
\partial_{\rho}=\left\{z \in L^{q} ;-\mathbb{E}(y z) \leq \rho(y), \forall y \in L^{p}\right\}
$$

the sub-gradient of $\rho$. We will assume that $\partial_{\rho}$ is convex and $\sigma\left(L^{q}, L^{p}\right)$-compact, $\dagger$ and

$$
\rho(y)=\max \left\{-\mathbb{E}(y z): z \in \partial_{\rho}\right\}
$$

holds for every $y \in L^{p}$. Furthermore, we will also impose that $z=1$ a.s. is in $\partial_{\rho}$,

$$
\partial_{\rho} \subset\left\{z \in L^{q} ; \mathbb{E}(z)=1\right\},
$$

and

$$
\partial_{\rho} \subset L_{+}^{q}=\left\{z \in L^{q} ; \mathbb{P}(z \geq 0)=1\right\} .
$$

Summarizing, we have the following.

Assumption 2.1: The set $\partial_{\rho}$ given by (1) is convex and $\sigma\left(L^{q}, L^{p}\right)$-compact, (2) holds for every $y \in L^{p}, z=1$ a.s. is in $\partial_{\rho}$ and (3) and (4) hold.

The above assumption is closely related to the Representation Theorem of Risk Measures stated by Rockafellar et al. (2006). Following their ideas, it is easy to prove that assumption 2.1 holds if and only if $\rho$ is continuous, and

(a) translation invariant, $\ddagger$

$$
\rho(y+k)=\rho(y)-k,
$$

for every $y \in L^{p}$ and $k \in \mathbb{R}$;

$\dagger \sigma\left(L^{q}, L^{p}\right)$-compact sets are also called weak ${ }^{*}$-compact sets. See Rudin (1973, p. 66) for further details.

$\$$ Some authors would say 'equation antivariant' rather than 'translation invariant'. 
(b) homogeneous,

$$
\rho(\alpha y)=\alpha \rho(y),
$$

for every $y \in L^{p}$ and $\alpha>0$;

(c) sub-additive,

$$
\rho\left(y_{1}+y_{2}\right) \leq \rho\left(y_{1}\right)+\rho\left(y_{2}\right),
$$

for every $y_{1}, y_{2} \in L^{p}$;

(d) mean dominating,

$$
\rho(y) \geq-\mathbb{E}(y),
$$

for every $y \in L^{p}$; and

(e) decreasing,

$$
\rho\left(y_{1}\right) \leq \rho\left(y_{2}\right)
$$

whenever $y_{1}, y_{2} \in L^{p}$ and $y_{1} \geq y_{2}$ a.s.

Particularly interesting examples are the conditional value at risk (CVaR) and the weighted conditional value at risk (WCVaR; Rockafellar et al. 2006), the dual power transform (DPT; Wang 2000), the Wang measure (Wang 2000) and the entropic value at risk (EVaR; Ahmadi-Javid 2012), among many others.

Remark 1: Fix $p \in[1,2]$ and consider the set $\mathcal{M}$ composed of those risk measures $\rho: L^{p} \longrightarrow \mathbb{R}$ satisfying assumption 2.1 (or, equivalently, continuous, translation invariant, homogeneous, sub-additive, mean dominating and decreasing). In addition, consider the set $\mathcal{S}$ composed of those subsets $\partial$ of $L^{q}(1 / p+1 / q=1)$ that are convex and $\sigma\left(L^{q}, L^{p}\right)$-compact, containing the constant random variable whose value is 1 , and such that, if $\partial \in \mathcal{S}$, then $\mathbb{E}(z)=1$ and $z \geq 0$ a.s. for every $z \in \partial$.

With the Hahn-Banach Separation Theorem (Rudin 1973, theorem 3.4, p. 58) and expressions (1) and (2), it is easy to prove that there is a one-to-one bijection,

$$
\begin{aligned}
\mathcal{M} & \leftrightharpoons \mathcal{S}, \\
\rho & \leftrightharpoons \partial_{\rho},
\end{aligned}
$$

between $\mathcal{M}$ and $\mathcal{S}$. This bijection is increasing, i.e. higher risk measures are associated with higher sets of $\mathcal{S}$. Consequently, given a finite family of risk measures satisfying assumption 2.1 ,

$$
\left\{\rho_{1}, \rho_{2}, \ldots, \rho_{k}\right\} \subset \mathcal{M}
$$

one can consider the family of sub-gradients

$$
\left\{\partial_{\rho_{1}}, \partial_{\rho_{2}}, \ldots, \partial_{\rho_{k}}\right\} \subset \mathcal{S} .
$$

Then, taking the convex hull

$$
\partial_{\rho}=\operatorname{Co}\left(\bigcup_{i=1}^{k} \partial_{\rho_{i}}\right),
$$

which is obviously $\sigma\left(L^{q}, L^{p}\right)$-compact, we easily prove that there exists $\rho$ satisfying assumption 2.1 such that $\rho_{i} \leq \rho$, $i=1,2, \ldots, k$. Furthermore, $\rho$ is the minimum element in $\mathcal{M}$ fulfilling this inequality.

\subsection{The pricing rule}

There are several ways to introduce pricing rules in a market with transaction costs (see, among many other interesting contributions, Jouini and Kallal (1995, 2001) or Schachermayer
(2004)). Nevertheless, they are all closely related and lead to quite similar assumptions. In line with the previous literature, we will consider the function

$$
\Phi: L^{2} \longrightarrow \mathbb{R}
$$

which provides us with the initial (at $t=0)$ price $\Phi(y)$ of a final (at $T$ ) pay-off $y \in L^{2}$. One can look at $y \in L^{2}$ as the random final wealth provided by a self-financing portfolio adapted to a given filtration (information arrival) $\left(\mathcal{F}_{t}\right)_{t \in[0, T]}$ with $\mathcal{F}_{0}=\{\emptyset, \Omega\}$ and $\mathcal{F}_{T}=\mathcal{F}$, whereas $\Phi(y)$ is the amount paid at $t=0$ by the investor.

Henceforth in this paper the words 'portfolio' and 'pay-off' will have the same meaning. We know that this is an abuse of terminology, since portfolios consist of linear combinations of assets. Nevertheless, note that every reachable pay-off $y \in L^{2}$ is the random final wealth provided by some self-financing portfolio adapted to information arrival (filtration). Thus, for $y$ we have a dynamic (and stochastic) portfolio paying $y$ at $T$. This portfolio is a linear combination of the available assets, although very probably it will have to be rebalanced. We believe that it improves the intuition to identify the pay-off $y$ and the stochastic portfolio generating this pay-off. In this paper this identification does not produce any confusion. $\dagger$

We will price securities in $L^{2}$ because we are assuming returns with finite variance. This property holds for the most important models of financial economics (CAPM, APT, Black and Scholes, stochastic volatility models, etc.) and is also supported by the empirical evidence (see, among other interesting contributions, Grabchak and Smorodnitski (2010)). We will also assume that every $y \in L^{2}$ has an initial price $\Phi(y)$, i.e. the market is complete. Most of the theory still holds if the domain of $\Phi$ is a closed subspace of $L^{2}$ (which implies that it is still a Hilbert space), but the exposition becomes much more complex. Needless to say, the most important frictionless pricing models of financial economics (Black and Scholes, stochastic volatility, etc.) are complete. $\$$

We will adopt the usual conventions for imperfect markets,

$$
\Phi\left(y_{1}+y_{2}\right) \leq \Phi\left(y_{1}\right)+\Phi\left(y_{2}\right),
$$

for every $y_{1}, y_{2} \in L^{2}$, and

$$
\Phi(\alpha y)=\alpha \Phi(y),
$$

for every $y \in L^{2}$ and every $\alpha>0$.

$\Phi(y)$ is usually interpreted as the ask price of $y \in L^{2}$, whereas $-\Phi(-y)$ is the bid price. Equation (6) leads to $\Phi(0)=$ 0 , since otherwise $\Phi(0)=\Phi(2 \times 0)=2 \Phi(0)$ would imply the contradiction $1=2$. Hence, inequality

$$
-\Phi(-y) \leq \Phi(y)
$$

follows trivially from (5). We will also assume that the lending rate is non-negative and not higher than the borrowing rate, i.e.

$$
0<-\Phi(-1) \leq \Phi(1) \leq 1
$$

must hold. Summarizing, we obtain the following.

$\dagger$ The authors sincerely thank the anonymous reviewer who pointed out the general necessity of making the distinction between a pay-off and the (perhaps dynamic) portfolio leading to this pay-off.

$\$$ Although 'formally' stochastic volatility models are not complete, in practice the existence of volatility-dependent assets is assumed. Otherwise it would be impossible to use the model so as to give a unique price of the available derivatives. 
Assumption 2.2: The pricing rule $\Phi: L^{2} \longrightarrow \mathbb{R}$ is continuous and satisfies (5), (6) and (8).

The following version of the Hahn-Banach Theorem is adopted from Rudin (1973, theorem 3.2, p. 56), and the proof is omitted since it is provided in that reference.

Theorem 2.3: Consider the linear subspace $L \subset L^{2}$ and the linear function $\varphi: L \longrightarrow \mathbb{R}$ such that $\varphi(y) \leq \Phi(y)$ for every $y \in L$. Then there exists $\phi: L^{2} \longrightarrow \mathbb{R}$ linear such that

$$
\phi(y)=\varphi(y),
$$

for every $y \in L$, and

$$
\phi(y) \leq \Phi(y),
$$

for every $y \in L^{2}$.

Corollary 2.4: The sub-gradient of $\Phi$, given by

$$
\partial_{\Phi}=\left\{z \in L^{2} ; \mathbb{E}(y z) \leq \Phi(y), \forall y \in L^{2}\right\},
$$

is convex and $\sigma\left(L^{2}, L^{2}\right)$-compact, and the expression

$$
\Phi(y)=\max \left\{\mathbb{E}(y z): z \in \partial_{\Phi}\right\}
$$

holds for every $y \in L^{2}$.

Proof: The convexity of $\partial_{\Phi}$ is trivial, so let us prove its weak $^{*}$-compactness. Since $\partial_{\Phi}$ is obviously weakly*-closed we only have to show that it is norm-bounded (Banach-Alaoglu's Theorem; see Rudin (1973, theorem 3.15, p. 66)). $\dagger$ The continuity of $\Phi$ implies the existence of $\delta>0$ such that

$$
\|y\| \leq \delta \Longrightarrow|\Phi(y)| \leq 1
$$

holds. Then

$$
\|y\| \leq \delta \Longrightarrow|\mathbb{E}(y z)| \leq 1, \quad \forall z \in \partial_{\Phi},
$$

holds, i.e.

$$
\|y\| \leq 1 \Longrightarrow|\mathbb{E}(y z)| \leq 1 / \delta, \quad \forall z \in \partial_{\Phi},
$$

holds. Expression (13) obviously implies that $\|z\| \leq 1 / \delta$ for every $z \in \partial_{\Phi}$.

Now let us see the fulfillment of (12). Obviously, it is sufficient to show the inequality $\Phi(y) \leq \max \left\{\mathbb{E}(y z): z \in \partial_{\Phi}\right\}$, where the maximum on the right-hand side is trivially attained due to the weak $^{*}$-compactness of $\partial_{\Phi}$. Fix $y_{0} \in L^{2}$ and the linear subspace generated by $y_{0}$, given by

$$
L=\left\{\lambda y_{0} ; \lambda \in \mathbb{R}\right\} .
$$

Consider the linear function $\varphi: L \longrightarrow \mathbb{R}$ given by

$$
\varphi\left(\lambda y_{0}\right)=\lambda \Phi\left(y_{0}\right),
$$

for every $\lambda \in \mathbb{R}$. The inequality $\varphi\left(\lambda y_{0}\right) \leq \Phi\left(\lambda y_{0}\right)$ is obvious from assumption 2.2 if $\lambda \geq 0$, and for $\lambda<0$, expressions (7) and (6) imply that

$$
\Phi\left(\lambda y_{0}\right) \geq-\Phi\left(-\lambda y_{0}\right)=\lambda \Phi\left(y_{0}\right)=\varphi\left(\lambda y_{0}\right) .
$$

Now consider the extension $\phi$ of $\varphi$ of theorem 2.3. The continuity of $\phi$ (assumption 2.2) implies the continuity of $\phi$. Indeed, given $\varepsilon>0$ there exists $\delta>0$ such that

$$
\|y\| \leq \delta \Longrightarrow|\Phi(y)| \leq \varepsilon
$$

$\dagger$ Recall that $L^{2}$ is reflexive and for a reflexive Banach space the closed unit ball is weakly (or weakly*)-compact. This property may be used instead of the Banach-Alaoglu Theorem. holds. Thus, (10) implies that

$$
\begin{aligned}
& \begin{array}{l}
\|y\| \leq \delta \Longrightarrow \\
\left\{\begin{array}{l}
\|y\| \leq \delta \Longrightarrow \phi(y) \leq \phi(y) \leq|\Phi(y)| \leq \varepsilon \\
\|-y\| \leq \delta \Longrightarrow-\phi(y)=\phi(-y) \leq \phi(-y) \leq|\Phi(-y)| \leq \varepsilon
\end{array}\right.
\end{array} \\
& \Longrightarrow|\Phi(y)| \leq \varepsilon .
\end{aligned}
$$

According to the Riesz Representation Theorem, take $z \in L^{2}$ with $\Phi(y)=\mathbb{E}(y z)$ for every $y \in L^{2}$. Then (10) shows that $z \in \partial_{\Phi}$, and (9) shows that $\mathbb{E}\left(y_{0} z\right)=\varphi\left(y_{0}\right)=\Phi\left(y_{0}\right)$.

Remark 2: For frictionless markets the set $\partial_{\Phi}$ contains a unique element usually called the stochastic discount factor (SDF). In our more general framework we will say that every element of $\partial_{\Phi}$ is a SDF of $\Phi$.

We will end this section by providing, without proof, a Mean Value Theorem. We will omit the proof because this is a particular case of several results regarding the representation of probability measures on convex sets by points. For instance, a more general proposition may be found in Phelps (2001, p. 3).

Henceforth, $\mathcal{C}\left(\partial_{\rho}\right)$ and $\mathcal{C}\left(\partial_{\Phi}\right)$ will denote the spaces of the real-valued $\sigma\left(L^{q}, L^{p}\right)$-continuous and $\sigma\left(L^{2}, L^{2}\right)$-continuous functions. $\mathcal{B}_{\rho}$ and $\mathcal{B}_{\Phi}$ will denote the Borel $\sigma$-algebras of $\partial_{\rho}$ and $\partial_{\Phi}$ endowed with topologies $\sigma\left(L^{q}, L^{p}\right)$ and $\sigma\left(L^{2}, L^{2}\right)$. $\mathcal{M}\left(\partial_{\rho}\right)$ and $\mathcal{M}\left(\partial_{\Phi}\right)$ will denote the Banach spaces of inner regular $\sigma$-additive measures on $\mathcal{B}_{\rho}$ and $\mathcal{B}_{\Phi} \cdot \mathcal{P}\left(\partial_{\rho}\right)$ and $\mathcal{P}\left(\partial_{\Phi}\right)$ will denote the subsets of $\mathcal{M}\left(\partial_{\rho}\right)$ and $\mathcal{M}\left(\partial_{\Phi}\right)$ composed of those measures that are probabilities (non-negative and total mass equal to 1). Recall that the Riesz Representation Theorem (Rudin 1973, theorem 6.19, p. 130) guarantees that $\mathcal{M}\left(\partial_{\rho}\right)$ and $\mathcal{M}\left(\partial_{\Phi}\right)$ are the dual spaces of $\mathcal{C}\left(\partial_{\rho}\right)$ and $\mathcal{C}\left(\partial_{\Phi}\right)$.

\section{Lemma 2.5: (Mean Value Theorem)}

(a) For every probability measure $m \in \mathcal{P}\left(\partial_{\rho}\right)$ there exists a unique $z_{m} \in \partial_{\rho}$ such that

$$
\int_{\partial_{\rho}} \mathbb{E}(y z) \mathrm{d} m(z)=\mathbb{E}\left(y z_{m}\right)
$$

holds for every $y \in L^{p}$.

(b) For every probability measure $m \in \mathcal{P}\left(\partial_{\Phi}\right)$ there exists a unique $z_{m} \in \partial_{\Phi}$ such that

$$
\int_{\partial_{\Phi}} \mathbb{E}(y z) \mathrm{d} m(z)=\mathbb{E}\left(y z_{m}\right)
$$

holds for every $y \in L^{2}$.

\section{Primal and dual optimal investment problems}

As stated in the Introduction, many classical financial problems involving decision making under risk have been revisited with risk measures (see Pflug and Römisch (2007) for a very complete study). Balbás et al. (2010) proposed a general optimal investment problem with coherent and expectation bounded risk measures and perfect pricing models. The natural extension for a market with friction is

$$
\left\{\begin{array}{l}
\min \rho(y), \\
\Phi(y) \leq 1, \quad \mathbb{E}(y) \geq R,
\end{array}\right.
$$

with $y \in L^{2}$ being the decision variable and $R>0$ representing the minimum required expected return. Problem (14) 
minimizes the risk of a pay-off $y$ whose global ask price is not higher than one dollar and whose expected value is at least $R$. Thus, it is a standard risk/return approach with $\rho$ as the risk measure.

Let us now give the conditions that guarantee that (14) is feasible (i.e. the feasible set is non-void).

Assumption 3.1: There exists $y_{1} \in L^{2}$ such that $0<\Phi\left(y_{1}\right)<$ $-\Phi\left(-\mathbb{E}\left(y_{1}\right)\right)$.

Assumption 3.1 is not at all restrictive, since it only imposes the existence of a portfolio $y_{1}$ whose expected return is higher than the borrowing rate. Indeed, suppose that an investor accepts a debt with value $\mathbb{E}\left(y_{1}\right)$ to be paid at $T$. Then he receives the bid price $-\Phi\left(-\mathbb{E}\left(y_{1}\right)\right)$ at $t=0$, which is higher than the price $\Phi\left(y_{1}\right)$ of $y_{1}$. Thus, he can buy $y_{1}$ and conserve the strictly positive quantity $-\Phi\left(-\mathbb{E}\left(y_{1}\right)\right)-\Phi\left(y_{1}\right)$, but his expected final wealth vanishes.

The proof of proposition 3.2 will show that assumption 3.1 may be relaxed slightly and (14) is still feasible. It is sufficient to impose the inequality

$$
\Phi\left(y_{1}-\mathbb{E}\left(y_{1}\right)\right)<0,
$$

although we believe that the given condition is more intuitive from a financial point of view.

Proposition 3.2: Under assumption 3.1, problem (14) is feasible for every $R>0$.

Proof: Obviously, portfolio $y_{1}-\mathbb{E}\left(y_{1}\right)$ has a negative price, since assumption 3.1 implies that

$$
\Phi\left(y_{1}-\mathbb{E}\left(y_{1}\right)\right) \leq \Phi\left(y_{1}\right)+\Phi\left(-\mathbb{E}\left(y_{1}\right)\right)<0 .
$$

Consider $k>0, R>0$ and portfolio

$$
x_{k, R}=k\left(y_{1}-\mathbb{E}\left(y_{1}\right)\right)+R \in L^{2} .
$$

One has

$$
\Phi\left(x_{k, R}\right) \leq k \Phi\left(y_{1}-\mathbb{E}\left(y_{1}\right)\right)+R \Phi(1) .
$$

Bearing in mind (15), we have

$$
k \geq \frac{1-R \Phi(1)}{\Phi\left(y_{1}-\mathbb{E}\left(y_{1}\right)\right)} \Longrightarrow \Phi\left(x_{k, R}\right) \leq 1 .
$$

In addition,

$$
\mathbb{E}\left(x_{k, R}\right)=k\left(\mathbb{E}\left(y_{1}\right)-\mathbb{E}\left(y_{1}\right)\right)+R=R .
$$

Therefore, $x_{k, R}$ is (14)-feasible if $k>0$ satisfies the lefthand side condition of (16).

Remark 3: Since (14) is feasible, hereafter $\rho_{R}^{*}$ will represent its optimal (infimum) value. Obviously, $\infty>\rho_{R}^{*} \geq-\infty$.

According to financial intuition, problem (14) should be bounded $\left(\rho_{R}^{*}>-\infty\right)$, and the optimal risk level $\rho_{R}^{*}$ should increase if the expected return $R$ does. We will deal with duality theory to analyse whether or not the above intuitive properties hold.

First, assumption 2.1 and corollary 2.4 allow us to substitute (14) by an equivalent problem. Consider problem

$$
\left\{\begin{array}{l}
\min \theta, \\
\theta+\mathbb{E}(y z) \geq 0, \quad \forall z \in \partial_{\rho}, \\
\mathbb{E}(y z) \leq 1, \quad \forall z \in \partial_{\Phi}, \\
\mathbb{E}(y) \geq R,
\end{array}\right.
$$

with $(\theta, y) \in \mathbb{R} \times L^{2}$ being the decision variable. It is easy to see that $y \in L^{2}$ solves (14) if and only if there exists $\theta \in \mathbb{R}$ such that $(\theta, y)$ solves (17), in which case $\theta=\rho(y)$ holds.

Assumptions 2.1 and 2.2 imply that (14) and (17) are convex problems, so we can use the general duality theory for convex optimization problems of Luenberger (1969, p. 223). Therefore, let us consider the Lagrangian function of (17)

$$
\begin{aligned}
& \mathbb{R} \times L^{2} \times \mathcal{M}\left(\partial_{\rho}\right) \times \mathcal{M}\left(\partial_{\Phi}\right) \times \mathbb{R} \ni\left(\theta, y, m_{1}, m_{2}, \lambda\right) \\
& \quad \rightarrow \mathcal{L}\left(\theta, y, m_{1}, m_{2}, \lambda\right) \in \mathbb{R},
\end{aligned}
$$

given by

$$
\begin{aligned}
\mathcal{L}(\theta, & \left.y, m_{1}, m_{2}, \lambda\right) \\
= & \theta\left(1-\int_{\partial_{\rho}} \mathrm{d} m_{1}\right)-\int_{\partial_{\rho}} \mathbb{E}\left(y z_{1}\right) \mathrm{d} m_{1}\left(z_{1}\right) \\
& +\int_{\partial_{\Phi}}\left(\mathbb{E}\left(y z_{2}\right)-1\right) \mathrm{d} m_{2}\left(z_{2}\right)+\lambda(R-\mathbb{E}(y)) .
\end{aligned}
$$

Then, $\left(m_{1}, m_{2}, \lambda\right) \in \mathcal{M}\left(\partial_{\rho}\right) \times \mathcal{M}\left(\partial_{\Phi}\right) \times \mathbb{R}$ is dual-feasible if and only if $m_{1} \geq 0, m_{1} \geq 0, \lambda \geq 0$, and $\mathcal{L}\left(\theta, y, m_{1}, m_{2}, \lambda\right)$ is bounded from below for $(\theta, y) \in \mathbb{R} \times L^{2}$, which obviously implies that $m_{1}\left(\partial_{\rho}\right)=1$, i.e. $m_{1} \in \mathcal{P}\left(\partial_{\rho}\right)$. Thus, the dual problem of (17) becomes

$$
\left\{\begin{array}{l}
\max \left(\operatorname { I n f } _ { y \in L ^ { 2 } } \left(-\int_{\partial_{\rho}} \mathbb{E}\left(y z_{1}\right) \mathrm{d} m_{1}\left(z_{1}\right)\right.\right. \\
\left.\left.+\int_{\partial_{\Phi}}\left(\mathbb{E}\left(y z_{2}\right)-1\right) \mathrm{d} m_{2}\left(z_{2}\right)+\lambda(R-\mathbb{E}(y))\right)\right), \\
\left(m_{1}, m_{2}, \lambda\right) \in \mathcal{P}\left(\partial_{\rho}\right) \times \mathcal{M}\left(\partial_{\Phi}\right) \times \mathbb{R} \\
m_{2}, \lambda \geq 0 .
\end{array}\right.
$$

However, bearing in mind lemma 2.5, and denoting $\mu=$ $m_{2}\left(\partial_{\Phi}\right)$, it is obvious that the above problem is equivalent to

$$
\left\{\begin{array}{l}
\max \left(\operatorname{Inf}_{y \in L^{2}}\left(-\mathbb{E}\left(y z_{1}\right)+\mu \mathbb{E}\left(y z_{2}\right)-\mu+\lambda(R-\mathbb{E}(y))\right)\right), \\
\left(z_{1}, z_{2}, \mu, \lambda\right) \in \partial_{\rho} \times \partial_{\Phi} \times \mathbb{R} \times \mathbb{R} \\
\mu, \lambda \geq 0
\end{array}\right.
$$

Since

$$
\begin{gathered}
-\mathbb{E}\left(y z_{1}\right)+\mu \mathbb{E}\left(y z_{2}\right)-\mu+\lambda(R-\mathbb{E}(y)) \\
=\mathbb{E}\left(y\left(-z_{1}+\mu z_{2}-\lambda\right)\right)-\mu+\lambda R,
\end{gathered}
$$

the infimum becomes greater than $-\infty$ if and only if $-z_{1}+$ $\mu z_{2}-\lambda$ vanishes, so the dual problem becomes

$$
\left\{\begin{array}{l}
\max -\mu+\lambda R, \\
z_{1}=\mu z_{2}-\lambda, \\
\left(z_{1}, z_{2}, \mu, \lambda\right) \in \partial_{\rho} \times \partial_{\Phi} \times \mathbb{R} \times \mathbb{R}, \quad \mu, \lambda \geq 0,
\end{array}\right.
$$

with $\left(z_{1}, z_{2}, \mu, \lambda\right) \in \partial_{\rho} \times \partial_{\Phi} \times \mathbb{R} \times \mathbb{R}$ being the decision variable.

Now let us prove that there is no duality gap between (14) and (19). $\dagger$

Theorem 3.3: Consider $R>0$. Under assumptions 2.1, 2.2 and 2.3, there is strong duality between (14) and (19), i.e. (14) is bounded if and only if (19) is feasible. In such a case, (19) is also bounded and solvable, and both optimal values coincide with $\rho_{R}^{*}>-\infty$.

Proof: Since (14) is equivalent to (17) and (19) is equivalent to (18), it is sufficient to prove that there is no duality gap

$\uparrow$ Note that there are several problems in mathematical finance leading to the existence of duality gaps. See, for instance, Jin et al. (2008). 
between (17) and (18). According to Luenberger (1969, theorem 1, p. 217), it is sufficient to prove that (17) satisfies the Slater Qualification, i.e. there exists $\left(\theta_{0}, y_{0}\right)$ satisfying all the constraints of (17) in terms of strict inequality. Fix $R>0$ and proposition 3.2 implies the existence of $y_{1}$ such that $\Phi\left(y_{1}\right) \leq 1$, $\mathbb{E}\left(y_{1}\right) \geq 4 R$. Then $y_{0}=y_{1} / 2$ satisfies $\Phi\left(y_{0}\right) \leq 1 / 2<1$ and $\mathbb{E}\left(y_{0}\right) \geq 2 R>R$. Therefore, (12) implies that $\mathbb{E}\left(y_{0} z\right) \leq$ $1 / 2<1$ for every $z \in \partial_{\Phi}$. Finally, choose

$$
\theta_{0}>\rho\left(y_{0}\right)=\max \left\{-\mathbb{E}\left(y_{0} z\right): z \in \partial_{\rho}\right\},
$$

and the first constraint of (17) will be strictly satisfied.

\section{The no-good-deal condition}

As already stated above, financial intuition indicates that problem (14) should be bounded, and its infimum value (the optimal risk level) should increase as does the expected return $R$. Let us show that these properties do not hold in general, unless there exists an appropriate SDF of $\Phi$.

\section{Lemma 4.1:}

(a) Under assumption 2.2, $-\Phi(-1) \leq \mathbb{E}(z) \leq \Phi(1) \leq 1$ holds for every $z \in \partial_{\Phi}$.

(b) Under assumptions 2.1, 2.2 and 2.3, if $\left(z_{1}, z_{2}, \mu, \lambda\right)$ is (19)feasible, then

$$
\frac{1+\lambda}{-\Phi(-1)} \geq \mu \geq \frac{1+\lambda}{\Phi(1)}
$$

\section{Proof:}

(a) Expressions (8) and (12) imply that

$$
\mathbb{E}(z) \leq \Phi(1) \leq 1
$$

and

$$
-\mathbb{E}(z) \leq \Phi(-1)
$$

(b) Bearing in mind (3), and taking expectations in the first constraint of (19), we have

$$
1=\mu \mathbb{E}\left(z_{2}\right)-\lambda \text {. }
$$

Thus, bearing in mind that $\mu \geq 0$, statement (a) leads to

$$
-\mu \Phi(-1)-\lambda \leq 1 \leq \mu \Phi(1)-\lambda,
$$

and (20) follows trivially.

Remark 4: Note that the (19)-feasible set does not depend on the required return $R>0$. If it is void, then theorem 3.3 shows that the optimal value of (14) becomes $\rho_{R}^{*}=-\infty$ for every $R>0$.

Remark 5: If the (19)-feasible set is not empty, then theorem 3.3 and (20) show that

$$
b_{R} \leq \rho_{R}^{*} \leq B_{R},
$$

where $b_{R}$ and $B_{R}$ are the optimal values of problems

$$
\left\{\begin{array}{l}
\max \frac{1+\lambda}{\Phi(-1)}+\lambda R=\frac{1}{\Phi(-1)}+\left(R+\frac{1}{\Phi(-1)}\right) \lambda, \\
z_{1}=\mu z_{2}-\lambda, \\
\left(z_{1}, z_{2}, \mu, \lambda\right) \in \partial_{\rho} \times \partial_{\Phi} \times \mathbb{R} \times \mathbb{R}, \quad \mu, \lambda \geq 0
\end{array}\right.
$$

and

$$
\left\{\begin{array}{l}
\max -(1+\lambda)+\lambda R=-1+(R-1) \lambda \\
z_{1}=\mu z_{2}-\lambda \\
\left(z_{1}, z_{2}, \mu, \lambda\right) \in \partial_{\rho} \times \partial_{\Phi} \times \mathbb{R} \times \mathbb{R}, \quad \mu, \lambda \geq 0
\end{array}\right.
$$

Bearing in mind (8), for

$$
R>\frac{1}{-\Phi(-1)}
$$

both problems have the same solution $\left(z_{1}^{*}, z_{2}^{*}, \mu^{*}, \lambda^{*}\right)$, which also solves

$$
\left\{\begin{array}{l}
\max \lambda \\
z_{1}=\mu z_{2}-\lambda \\
\left(z_{1}, z_{2}, \mu, \lambda\right) \in \partial_{\rho} \times \partial_{\Phi} \times \mathbb{R} \times \mathbb{R}, \quad \mu, \lambda \geq 0
\end{array}\right.
$$

Note that, according to theorem 3.3, problem (19) is bounded, and therefore (21) shows that (22) is bounded. Then (23) and (24) are also bounded, and (21) shows that the optimal value $\lambda^{*} \geq 0$ of (24) will satisfy

$$
\frac{1}{\Phi(-1)}+\left(R+\frac{1}{\Phi(-1)}\right) \lambda^{*} \leq \rho_{R}^{*} \leq-1+(R-1) \lambda^{*}
$$

for every $R>1 /[-\Phi(-1)]$.

Let us extend the notion of compatibility of Balbás et al. (2010) for models with transaction costs. Note that $\rho_{R}^{*}>-\infty$ holds for every $R>0$ if and only if it holds for some $R_{0}>0$, as indicated in remark 4 .

Definition 4.2: The couple $(\rho, \Phi)$ is said to be compatible if $\rho_{R}^{*}>-\infty$ or, equivalently, the (24)-feasible set is non-void. The couple $(\rho, \Phi)$ is said to be strongly compatible if there exists a (24)-feasible element $\left(z_{1}, z_{2}, \mu, \lambda\right)$ such that $\lambda>0$.

Remark 6: If $(\rho, \Phi)$ is not compatible, then we are facing a meaningless situation from a financial point of view. For every $R>0$ the optimal risk level becomes $-\infty$, so traders may construct pay-offs whose return is as large as desired and also whose risk is as small as desired. We will say that the value (risk, return $)=(-\infty, \infty)$ is available to investors.

If $(\rho, \Phi)$ is compatible, but it is not strongly compatible, then (25) shows that $\rho_{R}^{*} \leq-1$ for every $R>1 /[-\Phi(-1)]$, and therefore $\rho_{R}^{*} \leq-1$ for every $R>0$, since the (14)-feasible set obviously increases as $R>0$ decreases, and thus $\rho_{R}^{*}$ also decreases. Once again, traders may construct pay-offs with a risk level not higher than -1 and with the desired expected return. We will say that the value (risk, return) $=(-1, \infty)$ is available to investors.

In both cases we will say that there are good deals, which is unacceptable from a financial perspective.

Let us prove the main results of this paper, i.e. let us give the conditions that the SDF of $\Phi$ must satisfy so as to prevent the pathological existence of good deals. 


\section{Theorem 4.3:}

(a) If $\Phi$ satisfies assumptions 2.2 and 2.3 and for every SDF $z \in \partial_{\Phi}$ and every $\delta>0$ the inequality

$$
\mathbb{P}(z<\delta)>0
$$

holds, then $\Phi$ is not strongly compatible with any $\rho$ satisfying assumption 2.1.

(b) If $\Phi$ satisfies assumptions 2.2 and 2.3 and for any SDF $z \in \partial_{\Phi}$ and every $\delta>0$ the inequality

$$
\mathbb{P}(z \geq \delta)>0
$$

holds, then $\Phi$ is not compatible with any $\rho$ satisfying assumption 2.1 on the space $L^{1}$.

(c) If $\Phi$ satisfies assumptions 2.2 and 2.3, then there exists $\rho: L^{1} \longrightarrow \mathbb{R}$ satisfying assumption 2.1 and strongly compatible with $\Phi$ if and only if there exist $0<b \leq B$ and a SDF $z$ of $\Phi$ such that

$$
\mathbb{P}(b \leq z \leq B)=1 .
$$

(d) If $-\Phi(-1)=\Phi(1), \dagger \Phi$ satisfies assumptions 2.2 and 2.3 , and $\rho$ satisfies assumption 2.1, then $\Phi$ and $\rho$ are compatible if and only if there exists $\left(z_{1}, z_{2}\right) \in \partial_{\rho} \times \partial_{\Phi}$ such that $z_{2} / \Phi(1)$ is a linear convex combination of $z_{1}$ and the riskless asset $y=1$.

(e) If $-\Phi(-1)=\Phi(1)$, $\Phi$ satisfies assumptions 2.1 and 2.2, and $\rho$ satisfies assumption 2.1 , then $\Phi$ and $\rho$ are strongly compatible if and only if there exists $\left(z_{1}, z_{2}\right) \in \partial_{\rho} \times \partial_{\Phi}$ such that $z_{2} / \Phi(1)$ is a linear convex combination of $z_{1}$ and the riskless asset $y=1$ such that the weight of $y=1$ is strictly positive.

\section{Proof:}

(a) If $\Phi$ were strongly compatible with some $\rho$ satisfying assumption 2.1, then there would exist a (19)-feasible element $\left(z_{1}, z_{2}, \mu, \lambda\right)$ with $\lambda>0$. Then $z_{1}=\mu z_{2}-\lambda$ would imply that $\mu>0$, since otherwise $z_{1}=-\lambda<0$ would contradict (3). Consequently, bearing in mind (4),

$$
z_{2}=\frac{z_{1}+\lambda}{\mu} \geq \frac{\lambda}{\mu}
$$

and (26) does not hold for $z_{2}$ and $0<\delta<\lambda / \mu$.

(b) If $\Phi$ were compatible with some $\rho$, then there should exist a (19)-feasible element $\left(z_{1}, z_{2}, \mu, \lambda\right)$. As above, $\mu>0$, therefore

$$
z_{2}=\frac{z_{1}+\lambda}{\mu}
$$

Since $q=\infty$, we have that $z_{1}$ is essentially bounded, and therefore so is $z_{2}$. Thus (27) does not hold for $z_{2}$ if $\delta$ is large enough.

(c) The necessity of the given condition follows trivially from (a) and (b). It is also sufficient because one can choose the risk measure $\rho$ such that $\partial_{\rho}$ is the 'segment' $\left[z_{1}, 1\right]$, where

$$
z_{1}=\frac{(1+\lambda)}{\mathbb{E}(z)} z-\lambda
$$

(see remark 1, (8) and lemma 4.1(a)), and $\lambda>0$ is chosen so as to satisfy

$$
\frac{(1+\lambda)}{\mathbb{E}(z)} \geq \frac{\lambda}{b}
$$

$\dagger$ That is, if there is no friction when trading the riskless asset, or, equivalently, if lending rates equal borrowing rates.
Obviously, (3) and (4) hold, and the remaining conditions of assumption 2.1 become trivial. Expression (29) proves that

$$
\left(z_{1}, z, \frac{(1+\lambda)}{\mathbb{E}(z)}, \lambda\right)
$$

is (19)-feasible, and $\lambda>0$ implies that $(\rho, \Phi)$ is strongly compatible.

(d) If $\left(z_{1}, z_{2}, \mu, \lambda\right)$ is (19)-feasible, then lemma 4.1(b) implies that $\mu=(1+\lambda) / \Phi(1)$, so the constraint of (19) leads to

$$
\frac{z_{2}}{\Phi(1)}=\frac{z_{1}}{1+\lambda}+\frac{\lambda}{1+\lambda}
$$

Conversely, suppose that

$$
\frac{z_{2}}{\Phi(1)}=(1-t) z_{1}+t
$$

with $0 \leq t \leq 1$. If $t=1$, then $\left(z_{1}=1, z_{2}=\Phi(1), \mu=\right.$ $2 / \Phi(1), \lambda=1)$ is (19)-feasible. If $t \neq 1$, then

$$
z_{1}=\frac{1}{(1-t) \Phi(1)} z_{2}-\frac{t}{(1-t)},
$$

and

$$
\left(z_{1}, z_{2}, \mu, \lambda\right)=\left(z_{1}, z_{2}, \frac{1}{(1-t) \Phi(1)}, \frac{t}{(1-t)}\right)
$$

becomes (19)-feasible.

(e) Analogous to (d).

Let us provide some interpretation of the conditions of theorem 4.3. Artzner et al. (1999) interpreted the sub-gradient of their risk measures as a set of 'scenarios'. In other words, every $z_{1} \in \partial_{\rho}$ may be understood as a potential Radon-Nikodym derivative of some risk-neutral probability measure with respect to the probability measure $\mathbb{P}$. In such a case, the linear expression $L^{2} \ni y \longrightarrow \mathbb{E}\left(z_{1} y\right) \in \mathbb{R}$ may be interpreted as a possible (frictionless) pricing rule, and the global risk of $y$ is given by the maximum possible price of $y$ according to the above pricing rules (with the opposite sign; see (2)). In addition, (12) obviously implies that the same interpretation applies for imperfect pricing rules. Thus, since $z=1$ is in $\partial_{\rho}$ (assumption 2.1) and $\partial_{\rho}$ is convex, the condition of theorem $4.3(\mathrm{~d})$ is equivalent to the property $z_{2} / \Phi(1) \in \partial_{\rho}$. Hence, the compatibility of $(\rho, \Phi)$ requires the existence of "common scenarios' for $\rho$ and $\Phi$. Moreover, if we focus on theorem 4.3(e), strong compatibility requires something else, since the weight of $z=1$ cannot be zero and the common scenario $z_{2} / \Phi(1)$ cannot be 'in the border' of $\partial_{\rho}$. In some sense, a 'tangential intersection' of $\partial_{\rho}$ and $\partial_{\Phi} / \Phi(1)$ is not sufficient.

Remark 7: Theorem 4.3 implies the necessity of a SDF with a strictly positive lower bound, since otherwise the pathologies $($ risk, return $)=(-\infty, \infty)$ or $($ risk, return $)=(-1, \infty)$ will arise for every continuous, coherent and expectation bounded risk measure $\rho$. Moreover, these pathologies still hold if $\rho$ is replaced by a vector $\left(\rho_{1}, \rho_{2}, \ldots, \rho_{k}\right)$ and (14) becomes a vector optimization problem, i.e. for vector problems, one will obtain the solution

$$
\left(\text { risk }_{1}, \text { risk }_{2}, \ldots, \text { risk }_{k}, \text { return }\right)=(-1,-1, \ldots,-1, \infty) .
$$

Indeed, considering the risk measure $\rho$ of remark 1 , the inequality $\rho \geq \rho_{i}, i=1,2, \ldots, k$, holds, and the solution of 
$(14)$ is $($ risk, return $)=(-\infty, \infty)$ or $($ risk, return $)=(-1, \infty)$ if we use the risk measure $\rho$.

Remark 8: The existence of a SDF of $\Phi$ with a finite upper bound is also important, since otherwise the pathology (risk, return $)=(-\infty, \infty)$ will hold for every risk measure satisfying assumption 2.1 on the space $L^{1}$. There are many interesting examples, since Filipovic and Svindland (2012) have shown that $L^{1}$ is the natural space to define low-invariant convex risk measures. Concrete practical examples are, amongst others, the CVaR (also called the expected shortfall; see Kaina and Rüschendorf (2009), amongst others) and many versions of the WCVaR. Furthermore, the inequality VaR $\leq \mathrm{CVaR}$ for every level of confidence shows that the pathology (risk, return $)=(-\infty, \infty)$ will also hold for VaR.

Remark 9: It is worth pointing out that, for perfect markets, there is only one $\operatorname{SDF} z$, which must satisfy the existence of $0<b \leq B$ such that (28) holds so as to prevent the existence of good deals. An obvious implication is that a SDF with a log-normal distribution (for example, the Black and Scholes model; see Wang (2000) or Hamada and Sherris (2003)) or distributions with heavier tails (most of the stochastic volatility pricing models) will never be strongly compatible with any continuous, coherent and expectation bounded risk measure, and they will not be compatible with the VaR or with measures that can be extended to $L^{1}$. Thus, the already described pathologies will arise, i.e. good deals will be available to traders.

Remark 10: An important open question concerns the practical computation of good deals and their empirical performance. We will not address these topics, which are complex and require deep study clearly beyond the scope of this paper. Nevertheless, let us provide some general ideas that may help to construct good deals in practice.

First, the set of states $\Omega$ must be 'as small as possible', in order to guarantee that we can replicate the obtained good deal in practice. $\Omega$ should only contain those scenarios required to describe the price process. In this sense, for every price process (more generally, for every stochastic process) we can construct the probability space $(\Omega, \mathcal{F}, \mathbb{P})$ according to the DaniellKolmogorov Theorem (Kopp 1984, theorem 0.1.7, p. 7) which guarantees the absence of redundant or unnecessary scenarios. Furthermore, if some additional assumptions hold, then one can extend the $\sigma$-algebra $\mathcal{F}$ and the probability measure $\mathbb{P}$ according to the Prokhorov Theorem (Schwartz 1973, theorem 22, p. 81). In both cases the filtration $\left(\mathcal{F}_{t}\right)_{t \in[0, T]}$ may be selected (as usual in finance) as that generated by the price process. Obviously, the good deal (if it exists) will be a sequence in $L^{2}(\Omega, \mathcal{F}, \mathbb{P})$. However, since the set of simple functions is dense in this space, it may be very easily proved that the existence of good deals implies the existence of good deals composed of simple functions. Simple functions are given by

$$
\sum_{i} \alpha_{i} 1_{i}
$$

with every $\alpha_{i}$ being a scalar and every $1_{i}$ being an indicator function. Therefore, in finance, simple functions may be interpreted as a combination of digital (or binary) European options. Thus, one can construct good deals (when available) in a simple manner, since only digital options are sufficient. Needless to say, simple functions may be replicated in a complete market (see, for instance, Jarrow (2012) or Jarrow and Larsson (2012) for a formal definition of a complete market).

If we focus on a specific pricing model, then things are easily simplified. For instance, if we consider the (frictionless) Black and Scholes model, or a stochastic volatility model, then the set of states $\Omega$ may be the set of positive numbers $(0, \infty)$, since they are sufficient to represent the underlying asset price at $T . \mathcal{F}$ will be the Borel $\sigma$-algebra of $(0, \infty)$, and $\mathbb{P}$ will be the probability measure generated by a log-normal distribution (Black and Scholes) or that generated by the final price of the underlying asset (stochastic volatility). In this simple framework the SDF does not satisfy (28), and there are good deals available that can be constructed with simple functions, i.e. by combining digital options on the underlying asset. Obviously, once the combination of digital options has been computed by solving (14) or (19), the dynamic (stochastic) replication of this good deal will require a more complex probability space $(\Omega, \mathcal{F}, \mathbb{P})$, which will depend on the pricing model we are facing. In the Black and Scholes case the usual $\delta$-hedging is sufficient.

It may be interesting to see specific frictionless pricing models satisfying (28). Classical discrete-time pricing models (binomial, trinomial, etc.) may easily fulfill this expression, since the set $\Omega$ is finite and therefore every random variable is bounded. But things are quite different and much more complex for continuous-time pricing models. Indeed, denote by $z$ the unique element of $\partial_{\Phi}$, and assume that $z$ is the final (at $T$ ) value of the positive price process $\left(S_{t}\right)_{0 \leq t \leq T}$ of a self-financing portfolio adapted to some filtration $\left(\overline{\mathcal{F}}_{t}\right)_{0 \leq t \leq T}$ reflecting the arrival of information. It is not at all restrictive to assume that $\left(\log \left(S_{t}\right)-\log \left(S_{0}\right)\right)_{0 \leq t \leq T}$ is a Lévy process with stationary independent increments. Then, for every $n \in \mathbb{N}$, we have

$\log (z)=\log \left(S_{T}\right)=\log \left(S_{0}\right)+\sum_{i=1}^{n}\left(\log \left(S_{i T / n}\right)-\log \left(S_{[(i-1) T] / n}\right)\right)$,

and therefore $\log (z)$ will be given by the sum of independent variables with identical distribution. Hence, the Central Limit Theorem will make the fulfillment of (28) rather difficult. On the contrary, (26) and (27) will very often hold. Although this is only an intuitive approach rather than a formal proof, it may be useful to look for price processes $\left(S_{t}\right)_{0 \leq t \leq T}$ with adequate properties. Stationary (or independent) increments should be avoided, and the way to do that should be appropriate enough so as to reach a bounded random variable $S_{T}$. This is obviously a profound project that is beyond the scope of this paper.

\section{Cone constraints}

Some cone constraints affecting the pay-off $y$ might be useful to overcome the drawbacks pointed out in theorem 4.3 and its remarks for pricing rules without bounded SDF. Nevertheless, a quite natural constraint, such as the existence of lower bounds for the selected pay-off, does not impede the existence of good deals. Indeed, consider $M \in \mathbb{R}$ and assume that the constraint $y \geq M$ must hold. For some particular portfolio choice examples, if $M=0$, then we can interpret that there are short-selling restrictions. Problem (14) becomes

$$
\left\{\begin{array}{l}
\min \rho(y), \\
\Phi(y) \leq 1, \quad y \geq M, \quad \mathbb{E}(y) \geq R .
\end{array}\right.
$$


As in section 3, the dual problem becomes

$$
\left\{\begin{array}{l}
\max f(M)-\mu+\lambda R, \\
z_{1} \leq \mu z_{2}-\lambda, \\
\left(z_{1}, z_{2}, \mu, \lambda\right) \in \partial_{\rho} \times \partial_{\Phi} \times \mathbb{R} \times \mathbb{R}, \quad \mu, \lambda \geq 0,
\end{array}\right.
$$

with $f(M) \in \mathbb{R}$ depending on $M$. As in theorem 3.3, there is no duality gap between (30) and (31). As in the proof of theorem 4.3(a), if $\left(z_{1}, z_{2}, \mu, \lambda\right)$ is (31)-feasible and $\lambda>0$, then $\mu>0$ and

$$
z_{2} \geq \mu / \lambda>0,
$$

so one still needs a SDF with a strictly positive lower bound. Otherwise, the solution of (31) will be some $\left(z_{1}, z_{2}, \mu, \lambda=\right.$ $0)$ or $\left(z_{1}, z_{2}, \mu, \lambda=-\infty\right)$, and the optimal risk level will be $f(M)-\mu \leq f(M)$ or $-\infty \leq f(M)$, which does not depend on the required return $R$ and will be bounded from above. Thus, the caveat (risk, return) $=(f(M), \infty)$ will again apply. In particular, for perfect markets involving the lognormal or heavier-tailed distributions, (32) will not hold and the constraint $y \geq M$ (or $y \geq 0$ ) will not solve the caveat.

However, the existence of upper bounds for some SDF is no longer necessary, since the arguments in the proof of theorem 4.3(b) do not apply. The reason for this is that the first constraint in (31) is an inequality, instead of the equality of (19).

One could remove the constraint $\mathbb{E}(y) \geq R$, since $y \geq M$ imposes some kind of 'minimum achievement' in the investment. Then, the pay-off selection problem becomes

$$
\left\{\begin{array}{l}
\min \rho(y), \\
\Phi(y) \leq 1, \quad y \geq M
\end{array}\right.
$$
lem is

Suppose that (33) is feasible. As in section 3, the dual prob-

$$
\left\{\begin{array}{l}
\max f(M)-\mu \\
z_{1} \leq \mu z_{2} \\
\left(z_{1}, z_{2}, \mu\right) \in \partial_{\rho} \times \partial_{\Phi} \times \mathbb{R}, \quad \mu \geq 0
\end{array}\right.
$$

Once again there is no duality gap and (34) is solvable. These optimization problems are not very interesting because, obviously, (33) should not be feasible for $M$ large enough. Nevertheless, the lack of the $\lambda$ variable in (34) and the lack of equalities in the constraints of this problem imply that the proofs of theorems 4.3(a) and 4.3(b) no longer apply. Actually, the lack of a bounded SDF of $\Phi$ would not necessarily lead to any pathological property of (33). For instance, if $\rho=C V a R_{\alpha}$, $0<\alpha<1$ being the level of confidence, then (34) is often feasible, and therefore (33) is bounded and the optimal risk level will not be $-\infty$. Indeed, bearing in mind that

$$
\partial_{C V a R_{\alpha}}=\left\{z \in L^{\infty} ; 0 \leq z \leq \frac{1}{1-\alpha}, \mathbb{E}(z)=1\right\}
$$

(see Rockafellar et al. (2006), (8) and lemma 4.1(a)) it can easily be shown that if $z_{2}>0$ exists belonging to $\partial_{\Phi}$, then

$$
\left(z_{1}, z_{2}, \mu\right) \in \partial_{\rho} \times \partial_{\Phi} \times \mathbb{R}
$$

will be (34)-feasible for $\mu>0$ large enough.

\section{Conclusions}

In a recent paper, Balbás et al. (2010) proved that the usual frictionless pricing models (Black and Scholes, Heston, etc.) imply the existence of good deals (i.e. investors may construct pay-offs with (risk, return) values as close as desired to $(-\infty, \infty)$ or $(-1, \infty))$ for every continuous, coherent and expectation bounded measure of risk. It is natural to analyse whether the existence of friction may modify these findings that are obviously meaningless from a financial viewpoint.

This paper has addressed this caveat by considering a general pricing rule generating transaction costs, even when trading the riskless asset. Under general conditions concerning this pricing rule we have provided the necessary and the necessary and sufficient conditions that must hold so as to prevent the above pathology. These conditions do not depend on the concrete risk measure we are dealing with, and they mainly affect the pricing rule. The existence of a bounded SDF must hold, and the lower bound must be strictly positive. If there is no bounded SDF, or the lower bound is not greater than zero, then the above caveat will arise even for vector risk measures, and the existence of transaction costs will not solve the problem. It is worth remarking that a bounded SDF is not at all usual in the financial literature. In particular, for perfect markets, lognormal or heavier-tailed distributions for the SDF will imply the existence of good deals for every continuous, coherent and expectation bounded scalar or vector measure of risk.

\section{Acknowledgements}

This research was partially supported by RD_Sistemas SA, Welzia Management SGIIC SA, Comunidad Autónoma de Madrid, Spain (grant S2009/ESP-1594) and Ministerio de Economía, Spain (grants ECO2009-14457-C04 and ECO201239031-C02-01). The authors sincerely thank the anonymous reviewers and the participants at the Montreal Seminar on Actuarial and Financial Mathematics, whose comments led to improvements of this paper.

\section{References}

Ahmadi-Javid, A., Entropic value at risk: Anew coherent risk measure. J. Optimiz. Theory Applic., 2012, 155, 1105-1123.

Arai, T., Good deal bounds induced by shortfall risk. SIAM J. Financ. Math., 2011, 2, 1-21.

Artzner, P., Delbaen, F., Eber, J.M. and Heath, D., Coherent measures of risk. Math. Finance, 1999, 9, 203-228.

Aumann, R.J. and Serrano, R., An economic index of riskiness. $J$. Polit. Econ., 2008, 116, 810-836.

Balbás, A., Balbás, B. and Balbás, R., CAPM and APT-like models with risk measures. J. Bank. Finance, 2010, 34, 1166-1174.

Brown, D. and Sim, M., Satisfying measures for analysis of risky positions. Mgmt Sci., 2009, 55, 71-84.

Centeno, M.L. and Simoes, O., Optimal reinsurance. Revista de la Real Academia de Ciencias, RACSAM, 2009, 103, 251-264.

Cochrane, J.H. and Saa-Requejo, J., Beyond arbitrage: Good deal asset price bounds in incomplete markets. J. Polit. Econ., 2000, 108, 79-119.

Dimitrova, D.S. and Kaishev, V.K., Optimal survival reinsurance: An efficient frontier approach. Insurance: Math. Econ., 2010, 47, 27 35 .

Filipovic, D. and Svindland, G., The canonical model space for lawinvariant convex risk measures is $L^{1}$. Math. Finance, 2012, 22 585-589.

Föllmer, H. and Schied, A., Convex measures of risk and trading constraints. Finance Stochast., 2002, 6, 429-447. 
Foster, D.P. and Hart, S., An operational measure of riskiness. J. Polit. Econ., 2009, 117, 785-814.

Goovaerts, M., Kaas, R., Dhaene, J. and Tang, Q., A new classes of consistent risk measures. Insurance: Math. Econ., 2004, 34, 505516.

Goovaerts, M. and Laeven, R., Actuarial risk measures for financial derivative pricing. Insurance: Math. Econ., 2008, 42, 540-547.

Grabchak, M. and Smorodnitski, G., Do financial returns have finite or infinite variance? A paradox and an explanation. Quantit. Finance, 2010, 10, 883-893.

Guerra, M. and Centeno, M.L., Are quantile risk measures suitable for risk-transfer decisions? Insurance: Math. Econ., 2012, 50, 446461.

Hamada, M. and Sherris, M., Contingent claim pricing using probability distortion operators: Method from insurance risk pricing and their relationship to financial theory. Appl. Math. Finance, 2003, 10, 19-47.

Jarrow, R., The third fundamental theorem of asset pricing. Ann. Financ. Econ., 2012, 7, 1250007.

Jarrow, R. and Larsson, M., The meaning of market efficiency. Math. Finance, 2012, 22, 1-30.

Jin, H., Xu, Z.Q. and Zhou, X.Y., A convex stochastic optimization problem arising from portfolio selection. Math. Finance, 2008, 18, 171-183.

Jouini, E. and Kallal, H., Martingales and arbitrage in securities markets with transaction costs. J. Econ. Theory, 1995, 66, 178197.

Jouini, E. and Kallal, H., Efficient trading strategies in presence of market frictions. Rev. Financ. Stud., 2001, 14, 343-369.

Kaina, M. and Rüschendorf, L., On convex risk measures on $L^{p}$ spaces. Math. Meth. Oper. Res., 2009 (69), 475-495.

Kopp, P.E., Martingales and Stochastic Integrals, 1984 (Cambridge University Press: Cambridge).
Luenberger, D.G., Optimization by Vector Spaces Methods, 1969 (Wiley: New York).

Miller, N. and Ruszczynski, A., Risk-adjusted probability measures in portfolio optimization with coherent measures of risk. Eur. J. Oper. Res., 2008, 191, 193-206.

Pflug, G., and Römisch., 2007 (W: Modeling, Measuring and Managing Risk). (World Scientific: Singapore).

Phelps, R.R. Lectures on Choquet's Theorem, 2nd ed., 2001 (Lecture Notes in Mathematics 1757) (Springer: Berlin).

Rockafellar, R.T., Uryasev, S. and Zabarankin, M., Generalized deviations in risk analysis. Finance Stochast., 2006, 10, 51-74.

Rockafellar, R.T., Uryasev, S. and Zabarankin, M., Equilibrium with investors using a diversity of deviation measures. J. Bank. Finance, 2007, 31, 3251-3268.

Rudin, W., Functional Analysis, 1973 (McGraw-Hill: New York).

Rudin, W., Real and Complex Analysis, 3rd ed., 1987 (McGraw-Hill: New York).

Schachermayer, W., The fundamental theorem of asset pricing under proportional transaction costs in finite discrete time. Math. Finance, 2004, 14, 19-48.

Schwartz, L., Radon Measures on Arbitrary Topological Spaces and Cylindrical Measures, 1973 (Oxford University Press: Oxford).

Staum, J., Fundamental theorems of asset pricing for good deal bounds. Math. Finance, 2004, 14, 141-161.

Stoica, G. and Lib, D., Relevant mappings. J. Math. Anal. Applic., 2010, 366, 124-127.

Wang, S.S., A class of distortion operators for pricing financial and insurance risks. J. Risk Insur., 2000, 67, 15-36.

Zhiping, C. and Wang, Y., Two-sided coherent risk measures and their application in realistic portfolio optimization. J. Bank. Finance, 2008, 32, 2667-2673. 